\title{
Pathophysiological idiosyncrasies and pharmacokinetic realities may interfere with Tacrolimus dose titration in liver transplantation
}

Itziar Oteo ${ }^{1}$, John C Lukas ${ }^{1,2}$, Nerea Leal ${ }^{2}$, Elena Suarez ${ }^{1}$, Andres Valdivieso ${ }^{3,4}$, Mikel Gastaca ${ }^{3}$, Jorge Ortiz de Urbina ${ }^{3}$, Rosario Calvo ${ }^{1}$

${ }^{1}$ Department of Pharmacology, School of Medicine, Universidad del Pais Vasco, Spain

${ }^{2}$ Drug Modeling \& Consulting, Dynakin SL, Derio, Spain

${ }^{3}$ Liver transplantation unit, Cruces Hospital, Bilbao, Spain

${ }^{4}$ School of Medicine, Universidad del Pais Vasco, Spain

Keywords: Tacrolimus, liver transplantation, monitoring, pharmacokinetic assumptions, idiosyncrasy

\section{Corresponding author:}

Rosario Calvo

Department of Pharmacology

School of Medicine

Universidad del Pais Vasco

Leioa, 48940

Spain

+34946012761 (fixed)

+34946013220 (fax)

Email: rcalvo@ehu.es 


\section{ABSTRACT}

Purpose To explore the main factors that make difficult the empirical monitoring of Tacrolimus (TAC) in the early period post liver transplantation (LTx). Specifically, those aspects were stressed related with patient idiosyncrasy and clinical status and also those pharmacokinetic (PK) assumptions on which drug individualization is based in clinical practice.

Methods Retrospective monitoring data from 75 de novo liver transplant patients treated with twice daily TAC and followed for up to 15 days were analyzed. Extensive laboratory measures were available. Dose adjustment was done empirically using trough levels $\left(C_{\min }\right)$. The population was separated into two background subgroups for low or high values of AST, Group 1 and Group 2, respectively. Data during the first 4 Days post LTx were used for this grouping. Then, subgroups were identified for albumin (ALB) and hematocrit (HCT) both being either elevated (Group 1A) or reduced (Group 1B) based on cut-off's of 2.5 $\mathrm{g} / \mathrm{dl}$ and 28\%, respectively. Similarly for Group 2 into Group 2A and Group 2B for ALB and HCT being elevated or reduced, respectively.

Results The $\mathrm{C}_{\min }$ /Dose ratio (inversely proportional to $\mathrm{CL}$ ) had variability $(\mathrm{CV} \%>80 \%$ ) that was incongruently higher for the ratio than for $\mathrm{C}_{\min }$ and Dose separately. This was attributed to that most patients were not at steady state or physiologically stable early post LTx. Group 1 was more predictable than Group 2, which was responsible for the ratio variability. $C_{\min }$ was lower across reduced ALB and HCT patient groups, for similar AST conditions ( $1 \mathrm{~A}$ vs. $1 \mathrm{~B}$ and $2 \mathrm{~A}$ vs. $2 \mathrm{~B}$ ) likely due to increased TAC metabolic clearance $(C L)$ (reduced $C_{\min } /$ Dose). The same situation existed for $0-15$ Days post LTx and 4 - 15 Days post LTx observations. Group 2A was the main source of the paradoxical variability in $\mathrm{C}_{\min }$ /Dose (higher ratio of $2.7 ; \mathrm{CV}=100 \%$ ) suggesting lower clearance and difficulty in recovery of stability. In contract, Group 2B had the lower ratio $(1.4 ; 47 \%)$ but required the highest number of dose adjustments as it was hard to identify clinically. Group $1 \mathrm{~A}$ was the most predictable empirically. When using observations from 15 patients who entered the clinic in 2007 and 2008, the same sub-groups existed in the same proportions.

Conclusion The difficulty in empirical dose adjustment of TAC is associated to the inevitable noncompliance of PK assumptions early post LTx and also to the inherent complexity of the clinical condition leading to increased uncertainty for the clinician as to dose selection. Identifying these sub-categories provides a rational means of classifying patients akin to a phenotype. This complexity of the kinetics in LTx and TAC treatment does not invalidate $C_{\min }$ as a biomarker, but a Bayes algorithm including a full PK structure and these covariates would be optimal. 


\section{INTRODUCTION}

Published reports on the clinical optimization of Tacrolimus (TAC) therapy after solid organ transplantation (Tx) were deemed contradictory when a pan-European expert meeting took place in 2007 [1] Even at the meeting, lack of consensus centered mostly on the relationship between the measured blood trough concentration $\left(\mathrm{C}_{\mathrm{min}}\right)$ and the primary therapeutic effect (rejection avoidance), challenging the utility of $\mathrm{C}_{\min }$ for TAC dose individualization. The clinical observation that blood $\mathrm{C}_{\min }$ is correlated to efficacy as well as to adverse events has been a determining factor in the continued use of blood $\mathrm{C}_{\min }$ as a biomarker. The target $C_{\min }$ for efficacy and adverse events prevention was accorded to lie within 10 - 20 $\mathrm{ng} / \mathrm{mL}$ during the first month and can be reduced to $5-15 \mathrm{ng} / \mathrm{mL}$ in later stages or in differing clinical situations. Eventually, the clinical utility of therapeutic drug monitoring (TDM) was confirmed in TAC treatment but renewed research for appropriate markers and alternative strategies was recommended [1].

Therefore, the moment seems ripe to explore the practical pharmacokinetic (PK) difficulties that still exist in empirical achievement of predefined concentrations of TAC for a particular patient, especially in the early period post Tx. The situation is more peculiar in liver (LTX) since TAC is eliminated by the liver (only $1 \%$ of total body clearance is renal). In this case the liver is the transplanted organ itself hence the extensive variation in the PK with day post LTx (Day post LTx) [2-4]. TAC is highly bound to blood proteins resulting in very low unbound drug concentration (active form [5]) that is not routinely measured

in patients. TAC is also a substrate of CYP3A and P-glycoprotein (P-gp) both expressed in liver and in the gut with genetic specificity. Consequently, the systemic clearance $(C L)$ and the oral bioavailability $(F)$ vary widely leading to large variability in oral clearance $(\mathrm{CL} / \mathrm{F})$ and also in $t_{1 / 2}(8 \mathrm{~h}$ to over $100 \mathrm{~h})[6,7]$. On the other hand, biochemical variables (e.g. AST, ALT, GGT, Bilirubin (BILI)), surrogate markers of physiological status, clinical efficacy and safety, also vary with Day post LTx. These variables are also intrinsic covariates that can be altered by other intrinsic and extrinsic factors and consequently, something as intuitive as the relation between variability in $\mathrm{C}_{\min }$ /Dose and enzyme markers of hepatic function has not yet been established [7-9]. Due to the above complexities, an initial exhaustive analysis of the relation between the $\mathrm{PK}$ observations $\left(\mathrm{C}_{\min }, \mathrm{C}_{\min } / \mathrm{Dose}\right)$ and the laboratory variables with possible clinical significance could currently aid in interpreting the $\mathrm{C}_{\min }$ and establish how it could be used correctly for next dose adjustment.

It is also well known that the use of $\mathrm{C}_{\min }$ (as a biomarker) for adjusting the next dose empirically requires a series of PK assumptions and limitations that must carefully assessed before any decision is taken. The principal one being that $\mathrm{C}_{\min }$ is proportional to the patient drug exposure measured as area under the blood level versus time curve (AUC), generally under stable conditions. In the case of TAC this situation, due to its PK peculiarities, is only fulfilled at steady state (SS). In practice, due to the high variability in the half-life $\left(t_{1 / 2}\right)$, some patients may not be stable and thus undergo unnecessary dose changes.

Since individualization is crucial immediately post LTx, alternatives should be studied that based on $\mathrm{C}_{\min }$ do not require the assumption of steady state. Population PK models with covariates and Bayesian 
techniques have been useful in similar situations and applied by the authors among others [10] in therapy individualization. Although a considerable number of PK studies have been performed for TAC [2, 4, 11, 12-17], such individualization approaches are lacking. A possible reason could be that the covariates included in the models are selected across a non homogeneous array of variables and may not be justified physiologically, e.g. serum creatinine $[2,11,18]$.

Apparently, the ubiquitous lack of relation between $\mathrm{C}_{\min }$ and Dose in TAC LTx empirical monitoring needs to be further rationalized. In this study an exploration was performed of the observed trends in the $\mathrm{C}_{\min }$ and $\mathrm{C}_{\min } /$ Dose using retrospective data from hepatic transplant patients under TAC. The objective was to address the inherent difficulties in empirical TAC TDM by rationally integrating the sources of variability. The trends between relevant biochemical variables and PK criteria were explored in search of relationships that eventually included clinician anecdotal reports on physiological expectations. Finally, new insights are proposed here for future covariate structures in population models to be used in treatment individualization with TAC.

\section{METHODS AND DATA ANALYSIS}

\section{Patients and data collection}

Blood level observation data were collected retrospectively from routine monitoring records of 75 "de novo" adult patients receiving TAC (Prograf ${ }^{\circledR}$, Fujisawa Pharmaceutical, Osaka, Japan, now Astellas Pharma) after LTx in the period 1998-1999 at the Liver Unit Transplantation of the Cruces Hospital in Bilbao, Spain. Usage of the data was approved by the Medical Ethics Committee of the Cruces Hospital in Bilbao.

Immunosuppressive therapy with oral TAC dosing was initiated immediately after LTX at a single dose of $0.1 \mathrm{mg} / \mathrm{kg} / \mathrm{day}$. All patients were under similar doses of prednisone and 13 under azathioprine. The authors were at all times blinded regarding the true identity of these patients The patients were receiving TAC twice daily (a morning and an evening dose) starting on the LTx day (Day 0). Pre dose trough $\left(\mathrm{C}_{\min }\right)$ blood samples were collected immediately before the morning dose and recorded to the day of release from the hospital. Subsequent doses were adjusted empirically on the basis of clinical evidence of efficacy and toxicity aiming to maintain TAC $C_{\text {min }}$ between $10-20 \mathrm{ng} / \mathrm{mL}$. Dose adjustment was performed within the same day at the evening dose. Determination of TAC concentrations in blood samples at the hospital was performed using Microparticle Enzyme Immunoassay (MEIA), (Abbott Lab, Abbott Park, IL, USA). The method permits TAC determination in the range $1.5-30 \mathrm{ng} / \mathrm{mL}$. To date immunoassays are the most frequently used methods for TAC quantification.

The data collection focused on 0 - 15 Days post LTx. Relevant patient information was also extracted from the patient medical records files including demographic, laboratory variables and medication history. The dataset included TAC dose and $\mathrm{C}_{\min }$. Additionally, monitoring records were obtained from hepatic 
transplant patients $(2007,2008)$ treated with TAC BID. These observations were used to validate the grouping assumptions.

\section{Data analysis}

In a first step, an exploratory analysis of mean, standard deviation and coefficient of variation of all variables, including Day post LTx, was carried out. Graphical exploration and linear regression were used to test for relationship of $\mathrm{C}_{\min }$, Dose and $\mathrm{C}_{\min } /$ Dose $^{*}$ (Dose* is the previous daily dose) versus laboratory tests. Additionally, the interrelation between variables (i.e. AST and ALT) was also analyzed in order to eliminate components.

In a second step the population was first separated into two major groups (Groups 1 and 2) defined based on AST levels, during the first days. In order to avoid investigator subjectivity the following criterion was applied in an algorithmic selection (Table 1). Group 2 (elevated AST) was separated depending on fulfilling at least one of the following AST conditions: Day post $L T X=0$ AST $\geq 1000 \mathrm{U} / L$; Day post $L T x=1$ AST $\geq 750 \mathrm{U} / \mathrm{L}$; Day post $\mathrm{LTX}=2 \mathrm{AST} \geq 500 \mathrm{U} / \mathrm{L}$; Day post $\mathrm{LTX}=3$ AST $\geq 300 \mathrm{U} / \mathrm{L} ;$ Day post $\mathrm{LTX}=4$ AST $\geq$ $200 \mathrm{U} / \mathrm{L}$. To identify patients with worsening of liver function an additional criterion was applied as Day post LTx beyond day $4 \mathrm{AST} \geq 70 \mathrm{U} / \mathrm{L}$. The remaining patients composed Group 1 . From groups 1 and 2 two subgroups were distinguished for normal and sub normal Albumin (ALB) and HCT (combined), respectively. The following cut-offs were established: "ALB $\geq 2.5 \mathrm{~g} / \mathrm{L}$ and $\mathrm{HCT} \geq 28 \%$ " (Group $1 \mathrm{~A}, 2 \mathrm{~A}$ ), or "ALB $<2.5 \mathrm{~g} / \mathrm{L}$ and HCT $<28 \%$ " (Group 1B, 2B). These thresholds were established based on the criteria employed in practice.

S-Plus (Insightful Corp., Seattle, WA, USA) was used for data management, group selection and plots. The package SPSS (Chicago, IL, USA) was used for preliminary statistical testing.

Statistical tests were performed using the Student's t-test with a significance level at $p=0.05$. The correlation coefficient (Pearson's " $r$ ") and the linear regression coefficient $\left(r^{2}\right)$ were used for exploring relationships between variables.

\section{RESULTS}

Table 2 lists laboratory, treatment characteristics and the weight of the patients included in this study. From 0 to 15 days post LTx, 1113 records existed and 304 records contained both complete biochemistry and $C_{\min }$ values for an average of 5.6 occasions per patient. The maximum number of hospital stay days ranged from 9 to 15 Days post LTX.

All laboratory variables had high CV\% except ALB and HCT. The highest variability was associated with the biochemical tests related to hepatic function, and were dependent on Day post LTx e.g. the mean AST level varied from $498 \mathrm{U} / \mathrm{L}$ from 0 - 3 Day post LTx, to $64 \mathrm{U} / \mathrm{L}$ on $4-15$ Day post LTx. PK variables, $\mathrm{C}_{\min }$, Dose and mostly the $\mathrm{C}_{\min } /$ Dose* ratio showed large variability. The variability in the $\mathrm{C}_{\min } /$ Dose $^{*}$ ratio 
was $>80 \%$, higher than $\mathrm{C}_{\min }$ and Dose separately, and it did not improve in the $4-15$ Day post LTx period, supposedly more stable.

$\mathrm{C}_{\text {min }}$ showed significant correlation $(\mathrm{p}<0.05)\left(\right.$ but low $\left.\mathrm{r}^{2}\right)$ with AST, ALT, APH, Proteins, ALB and HCT but did not have any relation with BILI and urea or serum creatinine (the latter two used as indices of renal toxicity). The ratio $\mathrm{C}_{\min } /$ Dose $^{*}$ only showed a significant relationship with urea, creatinine and HCT but again with low $r^{2}$. Importantly, there was no correlation between $C_{\min }$ and Dose either.

In addition to the above variability, which is related to monitoring techniques, the inherent component of variation in TAC LTx was addressed explicitly. Based on physiological reasoning the population was separated depending on the influence of AST in $\mathrm{C}_{\min }$ as Group 1 ( $\left.\mathrm{n}=28\right)$ and Group 2 (elevated AST) ( $n=47$ ) (mean $77 \mathrm{U} / \mathrm{L}$ and $178 \mathrm{U} / \mathrm{L}, \mathrm{p}<0.05$, respectively) according to the criteria described in Methods. As expected $\mathrm{C}_{\min }$ was significantly higher in Group 2 at similar doses $(14.28 \mathrm{ng} / \mathrm{mL}$ vs. $12.45 \mathrm{ng} / \mathrm{mL}, \mathrm{p}<$ 0.05 ) at doses of $6.48 \mathrm{mg}$ and $6.35 \mathrm{mg}$, respectively. The variability in $C_{\min } /$ Dose was mostly associated to Group $2(92 \%)$, higher than $\mathrm{C}_{\min }(45 \%)$ and Dose (32\%), separately. This behavior was repeated in the 0 - 3 Day post LTx and 4-15 Day post LTx. Mainly in Group 2, a dichotomy was observed. Several patients had lower $\mathrm{C}_{\min }$ than that expected of high AST patients. Closer examination showed that those patients had lower levels of ALB and HCT. Therefore, these variables were selected for subgroup identification (Groups 1A, 1B and 2A, 2B). AST mean values were similar between Groups $1 \mathrm{~A}$ and 1B and also between Groups $2 A$ and 2B. Due to lack of sufficient measurements the $0-3$ Day post LTx sub group split was not possible.

Figure 1 depicts the final segregation of the population into four sub-groups (Table 1, Table 3). Day post LTx period was used as an additional separation variable, from $0-15$ Day post LTx (Panel A) and $4-15$ Day post LTx (Panel B). In Panel A (0 - 15 Days post LTx) it is seen that significantly higher doses were administered to Group $1 \mathrm{~B}$ than in Group $1 \mathrm{~A}$ but with a similar or lower $\mathrm{C}_{\min }$ in relation to its dose $\left(\mathrm{C}_{\mathrm{min}} /\right.$ Dose* was significantly less in Group $\left.1 \mathrm{~B}\right)$. Both Groups $1 \mathrm{~A}$ and $1 \mathrm{~B}$ showed coherent variability of $\mathrm{C}_{\min } /$ Dose* $^{*}$ (Table 3).

Group $2 \mathrm{~B}$ received similar doses to $2 \mathrm{~A}$, but its $\mathrm{C}_{\min }$ was significantly lower. The $\mathrm{C}_{\min } /$ Dose* was also less compared to $2 \mathrm{~A}$. In this last group the $\mathrm{C}_{\min } /$ Dose* $^{*}$ was highly unpredictable. For the $4-15$ Days post LTx period (Panel B) the situation was similar to that for $0-15$ Days post LTx. The variability in Group 2A (the highest) seems additionally associated to differential recovery of liver function. Two recovery rates for AST were evident in this group (that was associated to overall elevated AST during the first Days post LTx) (Figure 2). Group 2 required more dose changes than Group 1 (2.64 vs. 1.82, respectively) and Group 2B suffered the most changes (4.14). This is associated to the idiosyncrasy of these patients who have lower $\mathrm{C}_{\mathrm{min}} /$ Dose $^{*}$ (likely associated to higher clearance) than expected for their AST levels.

In a new group of 15 patients under TAC BID post LTX the same group characteristics and proportions were identified, validating the separation. The $\mathrm{C}_{\min } /$ Dose $^{*}(\mathrm{CV} \%)$ were $2.01(82 \%)$, respectively. Of the 15 
patients 6 (40\% of total) had elevated AST (Group 2) and $\mathrm{C}_{\min } /$ Dose $^{*}(\mathrm{CV} \%)$ of $2.53(83 \%)$. Additionally, 2 patients (13.3\%) belonged to Group 2B with $\mathrm{C}_{\min } / \mathrm{Dose}^{*}(\mathrm{CV} \%)$ of $1.36(54 \%)$.

\section{DISCUSSION}

In hospital practice, TAC dose individualization is carried out empirically, but this method is not efficient the first Days post LTx in all patients (multiple unnecessary dose changes) due to several critical issues some of which are discussed in this manuscript.

The first critical point presented here concerns the lack of significant linear correlation between TAC dosage and trough concentration $\left(C_{\min }\right)$, observed both in the literature $[19,20]$ and in the present study, directly impacting the (linear) causal flow dose - concentration - effect of TAC. Due to the above, monitoring of TAC has been based on the assumption that $C_{\min }$ correlated well with drug exposure (AUC), although the goodness of that relation depends on the level of stability. The second critical point regards the variability in the relation $C_{\min } /$ Dose. In this study, the $C_{\text {min }} /$ Dose was more variable than $C_{\min }$ or Dose alone. In this situation, $\mathrm{C}_{\min }$ /Dose cannot be considered a surrogate of $\mathrm{CL}$, which could explain why the ratio's connection with hepatic function is difficult to quantify while the literature is again contradictory on this issue $[21,22]$. Also paradoxically, $C_{\text {min }}$ did not correlate significantly with surrogates of adverse events (Creatinine, Urea) and had weak relation with surrogate indicators of effect (AST, ALT, APH and none with BILI). This is contrary to what is expected at steady state when relationships do exist. These singular situations can be associated with PK premises not taken into consideration.

A simple scheme can serve as demonstration of the above. Assuming elevated half-life $\left(t_{1 / 2}=40 \mathrm{~h}\right)$, after a $3 \mathrm{mg}$ BID dose, the steady state would be reached at $180 \mathrm{~h}$ with a $\mathrm{C}_{\min }$ of $18 \mathrm{ng} / \mathrm{mL}$ and $\mathrm{C}_{\min } /$ Dose $^{*}=3$. However, if a sample was taken on day $2\left(48 \mathrm{~h}\right.$ ), as is routine, the $C_{\min }$ would be $10.5 \mathrm{ng} / \mathrm{mL}$ (below the target in this case of $18 \mathrm{ng} / \mathrm{mL})\left(C_{\min } / D_{\text {Dose }}^{*}=1.75\right)$ and then, the dose would be increased to $4 \mathrm{mg} / 12 \mathrm{~h}$ for several days leading to levels above the target. Although a dose reduction was subsequently performed again to $3 \mathrm{mg} \mathrm{BID}$, due to accumulation, blood levels did not drop quickly and until day 15 the $\mathrm{C}_{\min }$ did not reach $18 \mathrm{ng} / \mathrm{mL}$. This value would have been achieved earlier with no dose changes if the initial treatment was kept until steady state.

In relation to the first point discussed in this paper and regarding clinical practice early post LTx, a considerable number of $\mathrm{C}_{\text {min }}$ measurements are performed before steady state is reached in some patients. In this situation, $\mathrm{C}_{\min }$ is influenced by the series of earlier doses and not only by the one immediately before the measurement. Depending on the $t_{1 / 2}$ and the dosing interval, the current $C_{\min }$ can be affected by more or less of the previous doses before steady state was reached. Theoretically, each dose change requires approximately 4 times the $t_{1 / 2}$ to reach the new steady state. Initial studies on the PK of TAC showed a $t_{1 / 2}$ of approximately 12 hours, hence the BID regimen for TAC. However in clinical practice of LTX, the $t_{1 / 2}$ varies widely, to the order of days. Then, empirical monitoring of TAC could be 
handicapped by fortuitous neglect of the assumption that the method is valid only when the sample is obtained at steady state $\left(\mathrm{C}_{\text {min_ss }}\right)$ in which case it is related to the $A \cup C[0-\infty], C L$ and the dose.

To avoid this problem other alternatives have been applied e.g. analyses of monitoring data at steady state $[2,4,22]$ or from the $7^{\text {th }}$ day onwards (assumed steady state) [16]. However, that is again based on approximations since (1) a priori it is not known what is the $t_{1 / 2}$ of a specific patient and (2) if the $t_{1 / 2}$ is long and steady state is reached after several days, monitoring is not performed when it is most needed i.e. at the beginning of treatment. Other sample time-points have also been explored such as $\mathrm{C}_{2}$ (concentration at 2 hours postdose), $C_{4}, C_{6}$ [22-25], but besides being better than $C_{\min }$, their clinical utility is scarce.

If the steady state assumption does not hold, $\mathrm{C}_{\min }$ is still a valid surrogate but alternative more complex methods should be used. Such methods include compartmental time-dependent PK models that provide predictions of concentrations after a certain Dose and a known CL. These methods require the use of specialized software. Population PK models represent the highest level of such efforts as they also provide the ability to subsequently use a Bayes prior to predict the individual patient PK [10]. Bayes prediction based on a population model with covariates, performed in a population with similar characteristics to the patient, is the optimum methodology because it does not require reaching steady state and provides individual PK parameters with only one value of $\mathrm{C}_{\min }$ after dose. However, it is not commonly applied with TAC possibly because it has not been possible to reach a consensus explaining the observed variability, in a convincing way to clinicians.

Thus, a key issue is the predictive ability of any method. For example, the existence or not of a predictive relationship between an apparently simple covariate, the patient's own body weight, and CL/F has not been established. Here, no significant relationship was found between weight and PK variables $\left(C_{\min }\right.$, Dose, $\mathrm{C}_{\min } /$ Dose $^{*}$ ) either. The disarray in published reports is even wider when searching for a relation between PK parameters and the actual clinical situation of the patient through biochemical laboratory test variables. Variable inclusion into a covariate model is typically performed in the same way as with any other continuous type covariate. But in the case of TAC in LTX, there are some unique issues involved. Laboratory values may change during the course of a study due to changes in liver function, other illness, the presence of other drugs, dietary changes or by simple random variation, to name just a few.

In the present study it was observed that there were two groups of patients with similar AST levels, receiving the same TAC dose but nevertheless had largely different and unexpectedly low blood levels. That could be due to the existence of subpopulations thus also explaining the lack of apparent relationship (or inconsistency) between $\mathrm{AST}$ and $\mathrm{C}_{\min }$ /Dose in population covariate analyses [9]. Further, the patients with lower $C_{\min }$ had elevated serum creatinine levels, which is paradoxical since renal toxicity is associated to elevated blood TAC levels. Now, $\mathrm{C}_{\min }$ /Dose had a significant tendency with urea and serum creatinine as in some population model reports [11, 16], but in others it is not included in the final model [18]. Anemia and hypoalbuminemia are frequently associated to renal insufficiency in hepatic transplant. This explains why some authors have paradoxically included serum creatinine as a covariate 
(although TAC has no renal excretion). Here, since creatinine, ALB, and HCT were correlated, the latter two were used as having better physiological relevance. Under that thinking eventually four groups were distinguished across ALB \& HCT, above or below $2.8 \mathrm{~g} / \mathrm{dL}$ and $25 \%$ (together), respectively and across AST reduction levels as described in the "Methods" section.

The groups with reduced HCT or ALB, Groups $1 B$ and $2 B$ (normal $A S T /$ reduced $A L B$ \& HCT and elevated $A S T /$ reduced ALB \& HCT) show lower $C_{\min }$ in relation to the dose and to the corresponding AST levels. If the reduced ALB \& HCT property were not identified, the clinician would encounter unexpectedly lower $\mathrm{C}_{\min }$ and would react by a dose increase. Although it is counter intuitive that patients with similar hepatic function could have higher CL/F, other authors report similar relations [15]. This behavior is possibly due to higher unbound fraction of circulating TAC secondary to reduced HCT and ALB [26]. Thus, given that TAC is a restrictive drug, Groups 1B, 2B could have higher drug flow through the liver for similar average intrinsic CL (related to enzymatic capacity) as Groups 1A, 2A. A rapid identification of Groups 1B and 2B can avoid unnecessary dose adjustments. In fact in this case study, Group 2B was less predictable and required the most dose changes compared to other groups. Interestingly, this pattern was maintained in newer patients with once again markedly lower $C_{\min } /$ Dose* in patients with reduced ALB \& HCT.

Regarding the identification here of sources of variability, it is mostly associated with Group 2A (elevated AST/ normal ALB \& HCT) in the period 4-15 Days post LTx. In this period there are two tendencies in AST: patients with rapid recovery or graft acceptance (between days 4 and 6) and patients with slow recovery. Knowledge of this behavior, a consequence of the PK of TAC and the type of transplant, could aid in individualizing the dose early on more efficiently (less adjustments). Importantly, this dichotomy can provide a physiological focus for covariate analysis based on rate of recovery (e.g. via AST) rather than post LTx time. Prospective experiments would be required for improved significance. A key parameter appears to be the slope of AST evolution from day 0 up to day 4 . It would be of interest to measure AST more frequently during that period.

Alternatives to blood level based individualization have been proposed such as monitoring of the pharmacodynamics or identification of the genetic pattern but, in the specific case of TAC in LTX, the information value of $\mathrm{C}_{\min }$ seems irreplaceable. Advances in pharmacogenetics have indeed created hope for explaining the interindividual variability in the PK of TAC. However, their application in the case of hepatic transplant has not yet reached a consensus [27] possibly due to the multiplicity of physiological processes concerned: Polymorphisms of the donor versus the recipient, changes in biochemical and clinical conditions across the treatment among others. Pharmacogenetic results are important for individualizing the first dose (Day post LTx $=0$ ) but in LTx the following doses must be adjusted on the basis of the patient-specific $\mathrm{C}_{\min }$ to also correct for - equally significant - non-genetics related variability. Further, with the introduction of a new once daily TAC formulation, Advagraf ${ }^{\circledR}$, the difficulty of an empirical 
adjustment may worsen due to prolonged duration of detectable levels in blood and delayed arrival to steady state (several days).

Clearly, the present study has some drawbacks in that the data are retrospective and hence not fully continuous. For example, there is scarcity of data during the critical first $36 \mathrm{~h}$ post LTx, key for establishing the AST evolution slope and similarly for the biochemical variables. Finally, the analytical assay, typical of such hospital units, may not be of laboratory precision. A prospective study could aim at improving on those aspects.

The present results suggest the investment of effort into integrating biochemical indicators and clinician reports of treatment efficacy as opposed to use of the $C_{\text {min }}$ alone. It seems that clinical utility of TAC TDM could improve with these considerations and so could covariate modeling efforts, preferably using prospective data. Clearly, during the first Days post LTx it is unavoidable to have patients outside steady state and with largely varying levels of hepatic functionality. Thus, the spotlight returns to the best known method for TDM i.e. via Bayes adjustment with user friendly specialized software packages. Usage of such methods, including improved covariate relations or patient categories such as the grouping performed here, can be of interest. 
Table 1 Four patient groups as defined by AST and ALB + HCT.

\begin{tabular}{|lcc|}
\hline & Normal ALB \& HCT ("A") & Reduced ALB \& HCT ("B”) \\
\hline Normal AST ("1") & Group 1A (All normal group) & Group 1B \\
\hline Elevated AST ("2") & Group 2A & Group 2B \\
\hline
\end{tabular}


Table 2 Demographic and laboratory variables and observed Dose and $C_{\min }$ post treatment for 75 patients from 0 to 15 Days post LTX.

\begin{tabular}{lcccc}
\multicolumn{1}{c}{ Variable } & Min & Max & Mean & CV (\%) \\
Demographic & & & & \\
Weight (kg) (Estimated) & 40 & 150 & 69.1 & $27 \%$ \\
& & & & \\
Laboratory tests & & & & \\
AST (U/L) & 9 & 4428 & 232.2 & $184 \%$ \\
ALT (U/L) & 6 & 3745 & 366.5 & $123 \%$ \\
GGT (U/L) & 13 & 1273 & 213.5 & $98 \%$ \\
ALP (U/L) & 78 & 1866 & 371.8 & $82 \%$ \\
Bilirubin (BILI) (mg/dL) & 0.3 & 41.8 & 3.8 & $126 \%$ \\
Prot (g/dL) & 3.5 & 7.8 & 5.7 & $12 \%$ \\
Albumin (ALB) (g/dL) & 1.9 & 6.2 & 3.0 & $12 \%$ \\
HCT (\%) & 19 & 47.6 & 29.7 & $14 \%$ \\
Urea (mg/dL) & 7 & 305 & 75.3 & $57 \%$ \\
Creatinine (mg/dL) & 0.4 & 4.3 & 1.2 & $41 \%$ \\
& & & & \\
Treatment & & & & \\
Max Day post LTx (days) & 9 & 15 & 14 & - \\
Dose (mg/day) & 1 & 15 & 6.39 & $32.71 \%$ \\
Cmin (ng/ml) & 1.5 & 30 & 13.61 & 47.24 \\
\& Cmin/Dose* (0 - 15 Day post LTx) & 0.3 & 23.10 & 2.32 & 82.76 \\
Cmin/Dose* (4 - 15 Day post LTx) & 0.36 & 23.10 & 2.34 & 89.53
\end{tabular}

$\S_{\text {In }} \mathrm{C}_{\min } /$ Dose $^{*}$, Dose ${ }^{*}$ stands for the dose immediately prior to the $C_{\text {min }}$ observation 
Table 3 Observed Dose, $\mathrm{C}_{\min }$ and $\mathrm{C}_{\min }$ /Dose post treatment for the four patient subgroups segregated by AST (1 and 2: normal and elevated) and ALB + HCT (A and B: normal and reduced) defined further in the text.

$\begin{array}{cccc} & \text { Period } & \text { Mean (range) } & \text { CV (\%) } \\ \text { DOSE (mg/day) } & & & \\ \text { Group 1A } & 0-15 & & \\ \text { Group 2A } & & 6.0(3-9) & 26 \\ \text { Group 1B } & 6.3(1-15) & 33 \\ \text { Group 2B } & 7.8(3-12) & 37 \\ & & 6.3(2-10) & 28 \\ \text { Group 1A } & 4-15 & & \\ \text { Group 2A } & & 6.1(3-9) & 26 \\ \text { Group 1B } & & 6.3(1-12) & 33 \\ \text { Group 2B } & & 6.7(4-12) & 37 \\ \text { G } & & \end{array}$

\section{$\operatorname{Cmin}(\mathrm{ng} / \mathrm{ml})$}

\begin{tabular}{|c|c|c|c|}
\hline & $0-15$ & & \\
\hline Group 1A & & $12.8(3.5-29)$ & 42 \\
\hline Group 2A & & $15.1(1.8-30)$ & 45 \\
\hline Group 1B & & $11.5(4.5-30)$ & 47 \\
\hline \multirow[t]{2}{*}{ Group 2B } & & $9.6(1.5-30)$ & 59 \\
\hline & $4-15$ & & \\
\hline Group 1A & & $12.8(5.1-22)$ & 31 \\
\hline Group 2A & & $14.6(1.8-30)$ & 41 \\
\hline Group 1B & & $10.8(4.5-16.4)$ & 34 \\
\hline Group 2B & & $8.5(4.6-20.2)$ & 44 \\
\hline \multicolumn{4}{|l|}{${ }^{\mathrm{C}} \mathrm{min} / \mathrm{Dose}{ }^{*}$} \\
\hline & $0-15$ & & \\
\hline Group 1A & & $2.3(0.9-6.7)$ & 48 \\
\hline Group 2A & & $2.6(0.4-23.1)$ & 92 \\
\hline Group 1B & & $1.7(0.4-3.7)$ & 50 \\
\hline \multirow[t]{2}{*}{ Group 2B } & & $1.6(0.3-4.3)$ & 56 \\
\hline & $4-15$ & & \\
\hline Group 1A & & $2.3(1-5.4)$ & 40 \\
\hline Group 2A & & $2.7(0.4-23.1)$ & 100 \\
\hline Group 1B & & $1.6(0.4-3.7)$ & 53 \\
\hline Group 2B & & $1.4(0.6-3.1)$ & 47 \\
\hline
\end{tabular}

$\S_{\text {In }} C_{\text {min }} /$ Dose ${ }^{*}$, Dose* stands for the dose immediately prior to the $C_{\text {min }}$ observation 


\section{Figure Legends}

Figure 1 Separation into patient groups of TAC Dose (open boxes) and $\mathrm{C}_{\min }$ (shaded boxes) first by AST (levels 1 and 2: normal and elevated) and then by ALB and HCT (levels A and B: normal and reduced). Panel A: Times 0 -15 Days post LTx; Panel B: Times 4 - 15 Day post LTx. The dotted lines simply join the means for Dose and $\mathrm{C}_{\min }$. Statistical comparisons for $\mathrm{C}_{\min }$, Dose and $\mathrm{C}_{\min } / \mathrm{Dose}^{*}$ are listed for groups of high or low starting AST.

Figure 2 Evolution of AST in 75 patients of Group 2A across 0 -15 Days post LTx (log expanded day scale) with two AST recovery rate patient subgroups (dotted lines). (Some patients appearing with normal levels on days $0-3$ worsened later on and hence were placed in this group.) 
Panel A

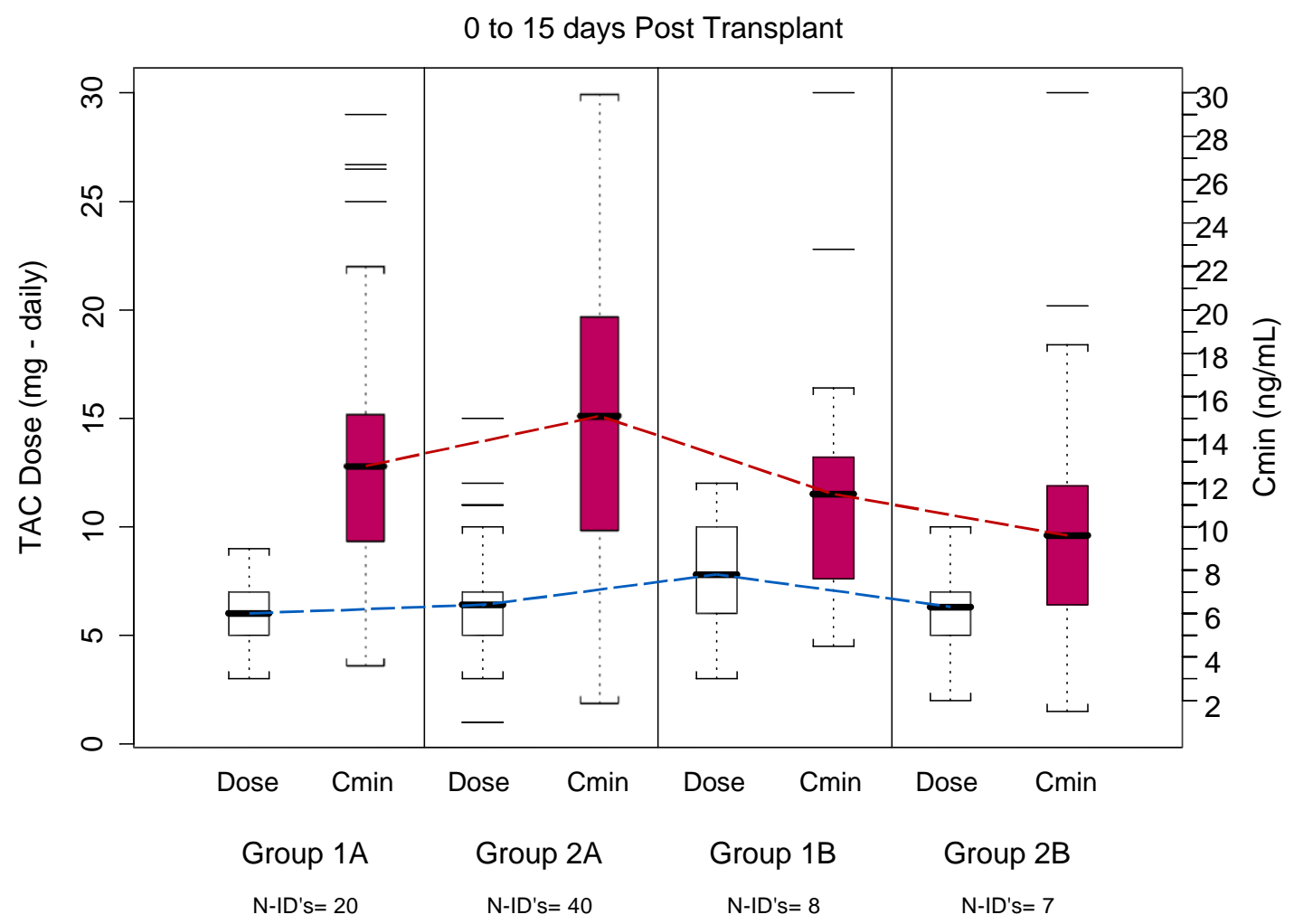

Dose: $1 \mathrm{~A}$ vs. $1 \mathrm{~B}: \mathrm{p}<0.05$; $2 \mathrm{~A}$ vs. $2 \mathrm{~B}: 0.90$,

$C_{\min }: 1 A$ vs. $1 B: p=0.27 ; 2 A$ vs. $2 B: p<0.05$,

$C_{\text {min }} /$ Dose*: $1 A$ vs. $1 B: p<0.05 ; 2 A$ vs. $2 B: p=0.25$ 


\section{Panel B}

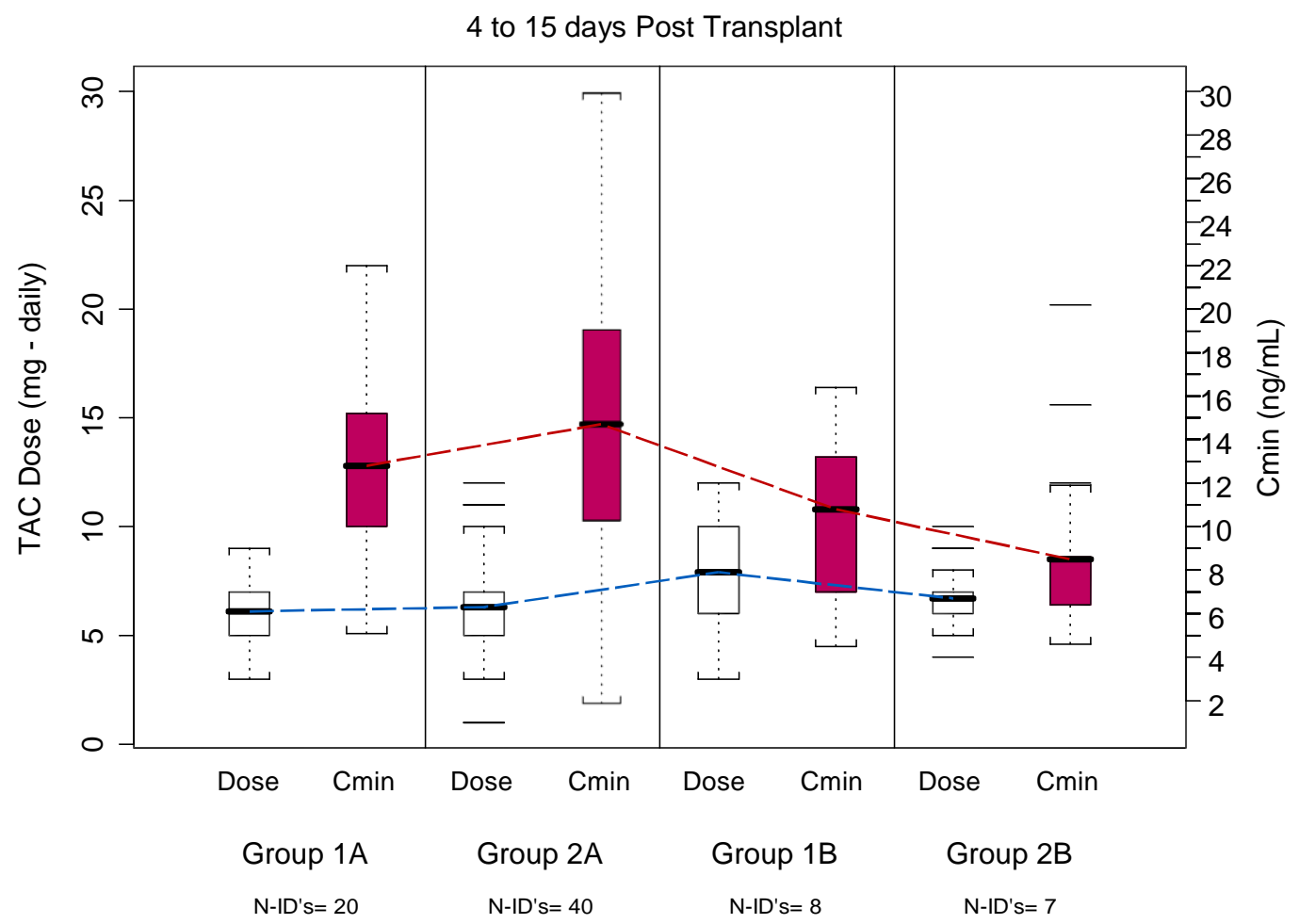

Dose: 1 A vs. $1 \mathrm{~B}: \mathrm{p}<0.05 ; 2 \mathrm{~A}$ vs. $2 \mathrm{~B}: \mathrm{p}=0.29$

$C_{\min }: 1 A$ vs. $1 B: p<0.05 ; 2 A$ vs. $2 B: p<0.001$

$\mathrm{C}_{\min } /$ Dose* $^{*}$ A vs. $1 \mathrm{~B}: \mathrm{p}<0.05 ; 2 \mathrm{~A}$ vs. $2 \mathrm{~B}: \mathrm{p}<0.001$ 


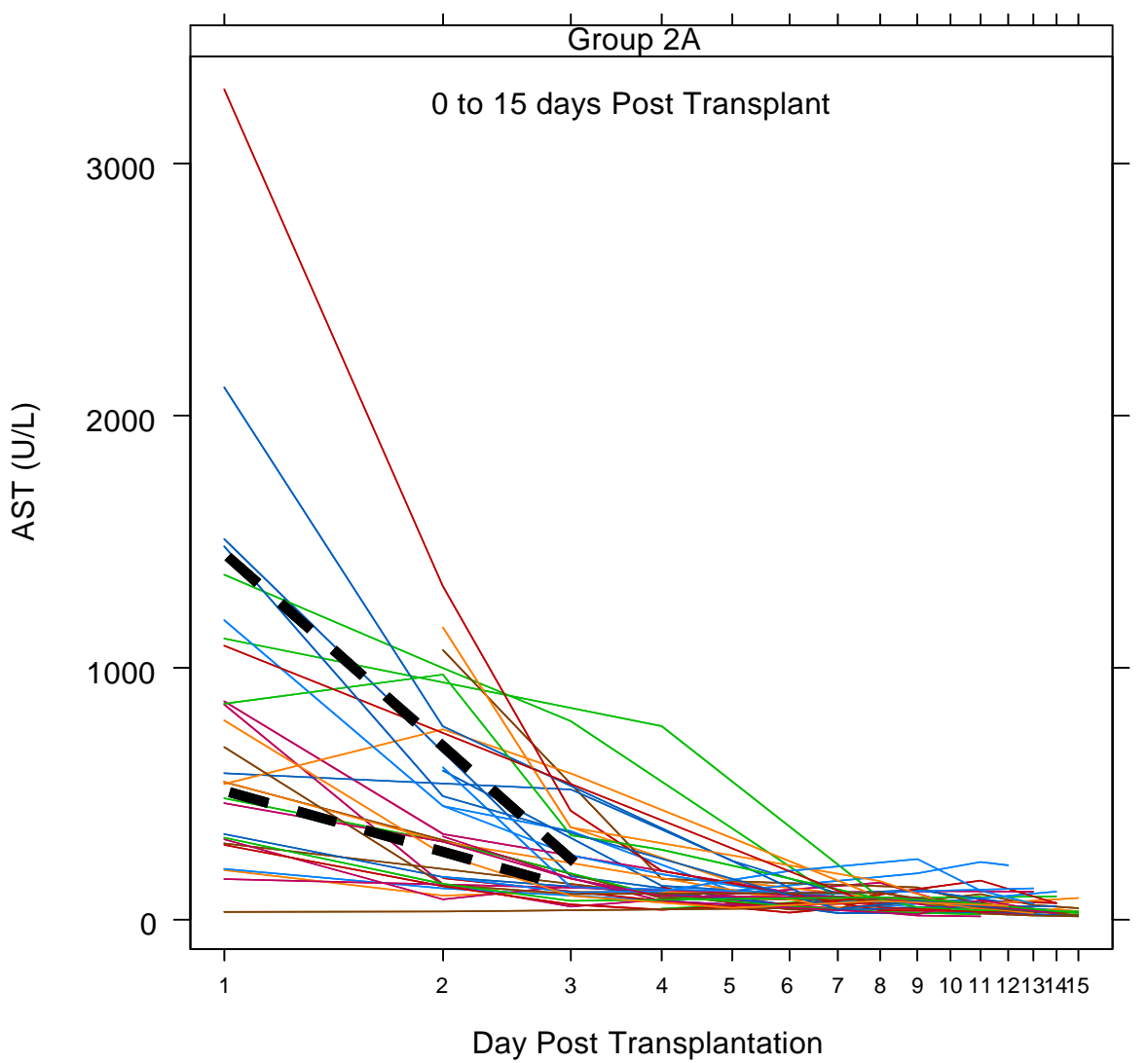




\section{References}

1. Wallemacq P, Armstrong VW, Brunet M, Haufroid V, Holt DW, Johnston A, et al (2009) Opportunities to optimize tacrolimus therapy in solid organ transplantation: report of the European consensus conference. Ther Drug Monit 31:139-152.

2. Antignac M, Hulot JS, Boleslawski E, Hannoun L, Touitou Y, Farinotti R, et al (2005) Population pharmacokinetics of tacrolimus in full liver transplant patients: modelling of the post-operative clearance. Eur J Clin Pharmacol 61:409-416

3. Fukudo M, Yano I, Masuda S, Goto M, Uesugi M, Katsura T, et al (2006) Population pharmacokinetic and pharmacogenomic analysis of tacrolimus in pediatric living-donor liver transplant recipients. Clin Pharmacol Ther 80:331-345

4. Lee JY, Hahn HJ, Son IJ, Suh KS, Yi NJ, Oh JM, et al (2006) Factors affecting the apparent clearance of tacrolimus in Korean adult liver transplant recipients. Pharmacotherapy 26:1069-1077

5. Zahir H, McCaughan G Gleeson M, Nand RA, McLachlan (2003) Factors affecting variability in distribution of tacrolimus in liver transplant recipients. Br. J. Pharmacol 57:298-309

6. Christians U, Jacobsen W, Benet LZ, Lampen A (2002) Mechanisms of clinically relevant drug interactions associated with tacrolimus. Clin Pharmacokinet 41:813-851

7. Staatz CE, Tett SE (2004) Clinical pharmacokinetics and pharmacodynamics of tacrolimus in solid organ transplantation. Clin Pharmacokinet 43:623-653

8. Böttiger Y, Brattström C, Tydén G, Säwe J, Groth CG (1999) Tacrolimus whole blood concentrations correlate closely to side-effects in renal transplant recipients. Br J Clin Pharmacol 48:445-448

9. Kuypers DR, Claes K, Evenepoel P, Maes B, Coosemans W, Pirenne J, et al (2004) Time-related clinical determinants of long-term tacrolimus pharmacokinetics in combination therapy with mycophenolic acid and corticosteroids: a prospective study in one hundred de novo renal transplant recipients. Clin Pharmacokinet 43:741-762

10. Karafoulidou A, Suarez E, Anastasopoulou I, Katsarou O, Kouramba A, Kotsi P, et al (2009) Population pharmacokinetics of recombinant factor VIII:C (ReFacto(R)) in adult HIV-negative and HIV-positive haemophilia patients. Eur J Clin Pharmacol 65:1121-1130

11. Fukudo M, Yano I, Shinsako K, Katsura T, Takada Y, Uemoto S, et al (2009) Prospective evaluation of the bayesian method for individualizing tacrolimus dose early after living-donor liver transplantation. J Clin Pharmacol 49:789-797

12. Jusko W, Piekoszewski, Klintmalm G, Shaefer M, Hebert M, Piergies A, et al (1995) Pharmacokinetics of tacrolimus in liver transplant patients. Clin Pharmacol Ther 57: 281-290.

13. Sam WJ, Aw M, Quak SH, Lim SM, Charles BG, Chan SY, et al (2000) Population pharmacokinetics of tacrolimus in Asian paediatric liver transplant patients. Br J Clin Pharmacol 50:531-541 
14. Macchi-Andanson M, Charpiat B, Jelliffe RW, Ducerf C, Fourcade N, Baulieux J (2001) Failure of traditional trough levels to predict tacrolimus concentrations. Ther Drug Monit 23:129-133

15. Zahir H, McLachlan AJ, Nelson A, McCaughan G, Gleeson M, Akhlaghi F (2005) Population pharmacokinetic estimation of tacrolimus apparent clearance in adult liver transplant recipients. Ther Drug Monit 27:422-430

16. Sam WJ, Tham LS, Holmes MJ, Marion AW, Quak SH, Lee KH, et al (2006) Population Pharmacokinetics of Tacrolimus in Whole Blood and Plasma in Asian Liver Transplant Patients. Clin Pharmacokinet 45:59-75

17. Blanchet B, Duvoux C, Costentin CE, Barrault C, Ghaleh B, Salvat A, et al (2008) Pharmacokineticpharmacodynamic assessment of tacrolimus in liver-transplant recipients during the early post $\mathrm{Tx}$ period. Ther Drug Monit 30:412-418.

18. Staatz CE, Willis C, Taylor PJ, Lynch SV, Tett SE (2003) Toward better outcomes with tacrolimus therapy: population pharmacokinetics and individualized dosage prediction in adult liver transplantation. Liver Transpl 9:130-137

19. Kahan BD, Koch SM (2001) Current immunosuppressant regimens: considerations for critical care. Curr Opin Crit Care 7:242-250

20. Venkataramanan R, Shaw LM, Sarkozi L, Mullins R, Pirsch J, MacFarlane G, et al (2001) Clinical utility of monitoring tacrolimus blood concentrations in liver transplant patients. J Clin Pharamacol 41:542-551

21. Undre NA, Schafer A (1998) Factors affecting the pharmacokinetics of tacrolimus in the first year after renal transplantation. European tacrolimus multicentre renal study group. Transplant Proc 30:12611263

22. Dansirikul C, Staatz CE, Duffull SB, Taylor PJ, Lynch SV, Tett SE (2006) Relationships of tacrolimus pharmacokinetic measures and adverse outcomes in stable adult liver transplant recipients. J Clin Pharm Ther 31:17-25

23. Holt D.W, Johnston A (2004) Monitoring Immunosuppressive Drugs. Has It a Future? Ther Drug Monit 26:244-247

24. Armendariz Y, Pou L, Cantarell C, Lopez R, Perello M, Capdevila L (2005) Evaluation of a limited sampling strategy to estimate area under the curve of tacrolimus in adult renal transplant patients. Ther Drug Monit 27:431-434

25. Wavamunno MD, Chapman JR (2008) Individualization of immunosuppression: concepts and rationale. Curr Opin Organ Transpl 13:604-608

26. Minematsu T, Sugiyama E, Kusama M, Hori S, Yamada Y, Ohtani H, et al (2004) Effect of hematocrit on pharmacokinetics of tacrolimus in adult living donor liver transplant recipients. Transplant Proc 36:1506-1511 
27. Staatz CE, Goodman LK, Tett SE (2010) Effect of CYP3A and ABCB1 Single Nucleotide Polymorphisms on the Pharmacokinetics and Pharmacodynamics of Calcineurin Inhibitors: Part II. Clin Pharmacokinet 49:207 - 221 